\title{
Effects of a Period of Asphyxia during Birth on Spatial Learning in the Rat
}

\author{
PATRICIA BOKSA, ANURADHA KRISHNAMURTHY, AND WILLIAM BROOKS \\ Douglas Hospital Research Centre, Departments of Psychiatry and of Neurology and Neurosurgery \\ McGill University, Montreal, Quebec, Canada
}

\begin{abstract}
The present study aimed to test whether an acute period of asphyxia during birth in the rat results in long-tcrm alterations in CNS function. Morphologic studics have indicated that the hippocampus is particularly vulnerable to perinatal anoxia. Thus, the present study tested adult rats, which had undergone acute birth asphyxia, for their performance in spatial learning and memory tasks associated with the hippocampus. Rat fetuses on the day of birth were submitted to an acute period of complete asphyxia by submersion of the isolated uterus into a water bath for 5-20 min before delivery of the pups. Control animals werc either born vaginally or delivered by rapid cesarean section. At $1.5 \mathrm{mo}$ of age, rats that had undergone $15 \mathrm{~min}$ of birth asphyxia showed no deficit in acquisition of spatial learning, measured as latency to find a hidden platform in the Morris water maze. However, at 4 mo of age, separate groups of rats, which had undergone 10, 15,
\end{abstract}

ABSTRACT
Recent epidemiologic studies indicate that asphyxia at or before birth remains an important problem in the general population and is particularly prevalent in the case of preterm neonates (1-3). Although perinatal asphyxia is thought to contribute to major deficits in some cases of mental retardation, cerebral palsy, and epilepsy $(1,2)$, a role for birth asphyxia in more subtle alterations of CNS functioning has been much less investigated. However, recent long-term follow-up studies have indicated that, for nondisabled school-aged children surviving moderate anoxic perinatal distress, mean psychoeducational test scores on several measures are significantly below those for the mild anoxic group or pecr controls (4).

During the birth process itself, mechanisms specific to the time of birth play an important protective role against hypoxic damage. In general, fetal animals are more resistant to hypoxia than are neonates $(5,6)$. Vannucci and Duffy $(7)$ have further shown that total cerebral energy consumption during anoxia, as well as consequent brain damage, is lower in fetal rats taken on the day of birth compared with neonates at $1 \mathrm{~d}$ of age, emphasizing the differences in physiology between the near-

Received April 26, 1994; accepted November 15, 1994.

Correspondence and reprint requests: Dr. P. Boksa, Douglas Hospital Research Centre 6875 LaSalle Blvd., Verdun, Quebec, Canada H4H 1R3.

Supported by a grant from the Medical Rescarch Council of Canada (P.B.) or $20 \mathrm{~min}$ of birth asphyxia, showed a deficit in initial acquisition of the spatial learning task compared with vaginally born controls, whereas the 5-min group performed similarly to controls. After overtraining, there was no difference among groups on short-term (1 wk) retention of the spatial navigation task; however, asphytic animals tested at 1.5 mo and retested at 4 mo showed a slight deficit in retention on retest. Animals that had undergone $15 \mathrm{~min}$ of birth asphyxia weighed less than did vaginally born animals, but showed no deficit in swimming ability, spontaneous alternation in a $\mathrm{T}$ maze, or other sensorimotor indices. Our results show that a brief period of asphyxia during a cesarean birth can produce subtle deficits in spatial learning that become evident only in adulthood. (Pediatr Res 37: 489-496, 1995)

term fetus and the same animal only $1 \mathrm{~d}$ later. Studies by Lagercrantz, Jones, Slotkin, and others (8-11) have indicated that the catecholamine surge elicited by a vaginal delivery serves to protect the fetus from hypoxia through redistribution of blood flow to heart, brain, and other vital organs, enhancement of lung compliance, and mobilization of glucose.

The notion that compensatory mechanisms involving the entire body are important in determining the extent of brain damage after birth asphyxia is also supported by cpidemiologic studies with human nconates. These studies indicate that CNS involvement occurs in less than half of human newborns who exhibit signs of intrapartum hypoxemia/acidosis (asphyxia) such as low Apgar scores, hypoxemia, acidemia, or asphyxial insult to other organ systems $(1,4)$. Even among a group of neonates classified as having suffered severe perinatal asphyxia often with acute multiple organ system effects, Shankaran et al. (12) have reported that a significant proportion (20\%) showed no signs of CNS involvement. Given the unique physiology of birth, including compensatory processes protecting against hypoxia/ischemia, it is of interest to determine the consequences, if any, of a period of asphyxia during this process.

Recently, Bjelke et al. (13) developed a model of asphyxia during a delayed cesarean section in the rat that involves removal of the entire uterus to a water bath, mimicking an acute asphyxial event, before delivery of the pups. Using this 
model, neuronal losses in areas CA1 and CA3 of the hippocampus have been observed in 3-wk-old animals that had undergone 14-17 min of birth asphyxia. Similarly, in fetal sheep subjected to carotid artery occlusion, hippocampal regions CA1, CA2, and CA3 are reported to be particularly vulnerable to hypoxic damage (14). These findings are in agreement with the frequent association of hypoxic/ischemic states in human neonates or adults with hippocampal lesions and memory deficits $(4,15,16)$.

Given this background, the first aim of this study was to delineate some of the long-term behavioral consequences of an acute period of asphyxia during birth in the rat. In light of the reported vulnerability of the hippocampus to perinatal anoxia, we chose to test rats that had undergone birth asphyxia for spatial learning in the Morris water maze, a test in which deficits are observed after dorsal hippocampal lesions (17-19). Several measures of sensorimotor function were also assessed. A second aim of the study was to examine time constraints surrounding behavioral deficits by $l$ ) determining the period of asphyxia necessary to produce deficits and 2) determining whether age at testing (adolescent versus adult) influcnces detection of deficits.

\section{METHODS}

Rats undergoing birth asphyxia during cesarean section delivery were generated using a modification of methods described by Bjelke et al. (13). Pregnant Sprague-Dawley rats at $22 \mathrm{~d}$ of gestation (i.e. on the day of birth) were decapitated and the entire uterus was quickly removed. Use of anesthetic in the dam was avoided because this compromised survival of the pups. Time taken for decapitation and removal of the uterus was less than $1 \mathrm{~min}$. An acute asphyxial episode was induced by completely immersing the uterus in a $37^{\circ} \mathrm{C}$ saline bath for $5,10,15$, or $20 \mathrm{~min}$. The pups were then delivered and breathing was stimulated by gentle tapping until breathing became even, usually within a few seconds; no other means of artificial resuscitation was used. Time between delivery of the first and final pups from a single uterus was approximately 1 min. Control pups were born vaginally; an additional group of pups delivered by cesarean section with no period of asphyxia was also included to control for the effects of the cesarean procedure alone, with no added hypoxia, on behavioral measures. Using this protocol, all pups born from a single dam were subjected to a single experimental condition (i.e. vaginal birth or cesarcan birth with $0,5,10,15$, or 20 min of asphyxia); therefore, to minimize interlitter effects, each experimental group was generated from at least three separate dams. Only male pups were retained for study. Pups were maintained for approximately $1 \mathrm{~h}$ on a heating pad, until placed with a foster dam. Groups of control and asphytic pups were identified by a coded pattern of toe-clipping and cross-fostered in mixed litters by the same dam to minimize differential rearing effects. Pups were weaned at $21 \mathrm{~d}$ and grown to $1.5,3$, or 4 mo of age, when behavioral testing was done. Before testing, individual animals were identificd by coded tail markings using water-insoluble marking pens, so that the experimental group (identified by toe-clipping) could be ignored during the behavioral testing.
Behavioral testing for spatial learning and memory was performed using the Morris water maze. In this task, animals use distal extra-maze cues to find a platform hidden beneath the water surface in a swimming pool. The water maze was a circular pool, $136 \mathrm{~cm}$ in diameter, filled with opaque milky water at $29^{\circ} \mathrm{C}$ and placed in a room with salient extra-maze cues. A plexiglass platform (10 cm square) was hidden $4 \mathrm{~cm}$ below the water surface in the center of one quadrant and remained in the same quadrant, except where indicated. Each trial of the spatial learning task was started by placing a rat with its face toward the wall of the pool at one of four quasi-randomly selected start points around the perimeter of the pool (north, south, east, or west). The trial was terminated when the animal located the platform and remained on it for 10 $\mathrm{s}$ or after $120 \mathrm{~s}$ if the rat failed to reach the platform. Two trials per day with an intertrial interval of 15 min were given on 8 consecutive d. Because two start points were proximal to the platform and two were distal, each daily test comprised one trial with a proximal start point and one with a distal start point. Latency to find the platform and swim paths were recorded. After the second trial on the eighth test day, 4-mo-old animals were given a probe trial consisting of placing the rat in the pool for $60 \mathrm{~s}$ with the platform removed; time spent in each quadrant of the pool was recorded. After this, randomly selccted subgroups of 4-mo-old animals reccived $2 \mathrm{~d}$ of massed training and 1 wk later were retested for short-term retention of the spatial learning task. Massed training consisted of eight trials per day with the platform in place, and the animal was allowed to remain on the platform for $30 \mathrm{~s}$ between each trial. Shortterm retention tests consisted of two trials per day for $4 \mathrm{~d}$ with an intertrial interval of $15 \mathrm{~min}$.

Spontaneous alternation was monitored in a $\mathrm{T}$ maze, consisting of a $90-\mathrm{cm}$ long arm and two $68-\mathrm{cm}$ choice arms. On each of two trials, the animal was placed at the distal end of the long arm, and a choice of left or right was recorded when the front paws crossed a line $21 \mathrm{~cm}$ into the choice arm. A trial was terminated when the animal exited the choice arm or remained in the arm for a maximum of $20 \mathrm{~s}$ or, if no choice was made, after $80 \mathrm{~s}$. Animals received 15 pairs of trials over $7 \mathrm{~d}$.

Sensorimotor performance was measured once daily for $5 \mathrm{~d}$ using tests modified from Dunnett et al. (20), Schallert et al. (21), and Welner and Koty (22). In brief, these tests included the following.

Tactile stimulation. Response to tactile stimulation was assessed by placing a circular adhesive label on the rat's snout in the center of the triangle made by the two eyes and nose. Latencies to begin grooming and to remove the label were recorded.

Orientation. The animal's vibrissac were lightly stroked with a cotton swab on the left side and then on the right. Turning of the head toward the stimulus was given a score of 0, whereas no response was given a score of 1 .

Head scans. The animal was placed on a circular metal stand of diameter $29 \mathrm{~cm}$ and height $40 \mathrm{~cm}$ for $60 \mathrm{~s}$. A rat will normally explore with dorsal, ventral, and lateral head scans, which consist of lifting its head upward, lowering its head over the side of the stand, and moving its head side to side, respectively. An animal that exhibited at least one dorsal head 
scan was given a score of 0 , whereas an animal that did not received a score of 1 ; ventral and lateral head scans were similarly scored.

Limb clasping. The animal was gently lifted by the base of the tail for a few seconds and deviations in the positioning of its fore- and hindlimbs from the normal (i.e., forepaws forward and hindpaws up and to the rear) were scored as 1,2, or 3 for limb deviations of $45^{\circ}, 90^{\circ}$, or $135^{\circ}$, respectively, toward the body from the normal position. A score of 4 was given if the limbs were clasped together or to the body.

Limb placing. The animal was held by the tail and gently placed onto the laboratory counter and the position of its forelimbs was observed. Scores of 0,1 , or 2 were given if the paws were extended either before contact with the surface, only after the vibrissae touched the surface, or not at all.

Turning on grid. The animal was placed with its head facing downward on a grid inclined $40^{\circ}$ from the horizontal, and the latency for the animal to right itself to face upward was recorded.

Statistical analyses of swim maze data were performed using a two-way analysis of variance with repeated measures or, for separate comparisons on individual test days, a one-way analysis of variance. When warranted by significant $F$ ratios, individual comparisons were made with post hoc Duncan's tests.

\section{RESULTS}

Survival rates were $100 \%$ for vaginally born animals and animals undergoing 0,5 , or $10 \mathrm{~min}$ of birth asphyxia, were $92 \%$ after $15 \mathrm{~min}$ of asphyxia and dropped to $40 \%$ with $20 \mathrm{~min}$ of asphyxia in the absence of any form of artificial ventilation. During the first few minutes after birth, animals undergoing 15 min of birth asphyxia lacked muscle tone in comparison with control animals (vaginally born or cesarean section with 0 min of asphyxia), took longer to initiate breathing, and had a reduced respiratory rate. Adult animals that had undergone 0-20 min of birth asphyxia did not exhibit any overt behavioral deficits. At 44 and $82 \mathrm{~d}$ of age, vaginally born animals weighed significantly more than did animals that had undergone $15 \mathrm{~min}$ of birth asphyxia; at $82 \mathrm{~d}$ of age, vaginally born animals also weighed more than animals delivered by cesarean section with 0 min of asphyxia (Table 1).

Spatial learning in adult animals that had undergone birth asphyxia was tested using the Morris water maze. At 4 mo of age, rats that had been vaginally born or born by cesarean section with $0,5,10,15$, or 20 min of birth asphyxia were

Table 1. Effect of birth asphyxia on body weight*

\begin{tabular}{lcc}
\hline & \multicolumn{2}{c}{ Weight $(\mathrm{g})$ at age } \\
\cline { 2 - 3 } & \multicolumn{1}{c}{$44 \mathrm{~d}$} & $82 \mathrm{~d}$ \\
\hline Vaginal birth & $201 \pm 5(11)$ & $399 \pm 11(11)$ \\
0-min asphyxia & $187 \pm 6(12)$ & $350 \pm 7 \dagger(11)$ \\
15-min asphyxia & $178 \pm 5 \dagger(8)$ & $345 \pm 17 \dagger(10)$ \\
\hline
\end{tabular}

* $F$ ratios for the main effect of birth group were $F(2,28)=4.30, p<0.03$ at $44 \mathrm{~d}$ of age and $F(2,29)=6.50, p<0.005$ at $82 \mathrm{~d}$ of age. $n=$ numbers in parentheses.

$\dagger p<0.01$, difference from vaginal birth value. tested on 8 consecutive $\mathrm{d}$ for latency to find a hidden platform in the Morris water maze (Fig. $1 a$ and $b$ ). Analysis of variance showed a significant effect of group $[F(5,144)=2.72, p<$ $0.02]$ and of day $[F(7,1008)=77.79, p<0.00001]$ but no interaction $[F(35,1008)=1.12, p=0.29]$. Separate comparisons based on each group's latencies across the $8 \mathrm{~d}$ showed that the 15- and 20-min asphyxia groups had an increased latency to find the platform in comparison with the vaginally born animals $(p<0.05)$. Although there was no significant group $\times$ time interaction, inspection shows that most of the differences in the groups occurred on $\mathrm{d} 2-7$. The groups did not differ on $\mathrm{d} 1$, and all groups eventually learned the task effectively by $\mathrm{d} 8$. When latencies for each day were considered separately, significant $(p<0.05)$ increases in latency, compared with the vaginally born group, were found on $\mathrm{d} 4$ and 5 for the 10-min asphyxia group, on d 2 and 4 for the 15-min asphyxia group and on d 4 and 6 for the 20-min asphyxia group. Significantly $(p<0.05)$ longer latencies were also observed on $\mathrm{d} 2$ for the 15 -min asphyxia group in comparison with either the 0 -min or the 5-min asphyxia groups, on d 5 for the 10-min asphyxia group compared with the 5-min group, and on $\mathrm{d} 7$ for the 20-min asphyxia group compared with either the 0 -min or the 5 -min groups. Adult rats delivered by cesarean section with no asphyxia (0-min asphyxia) or with
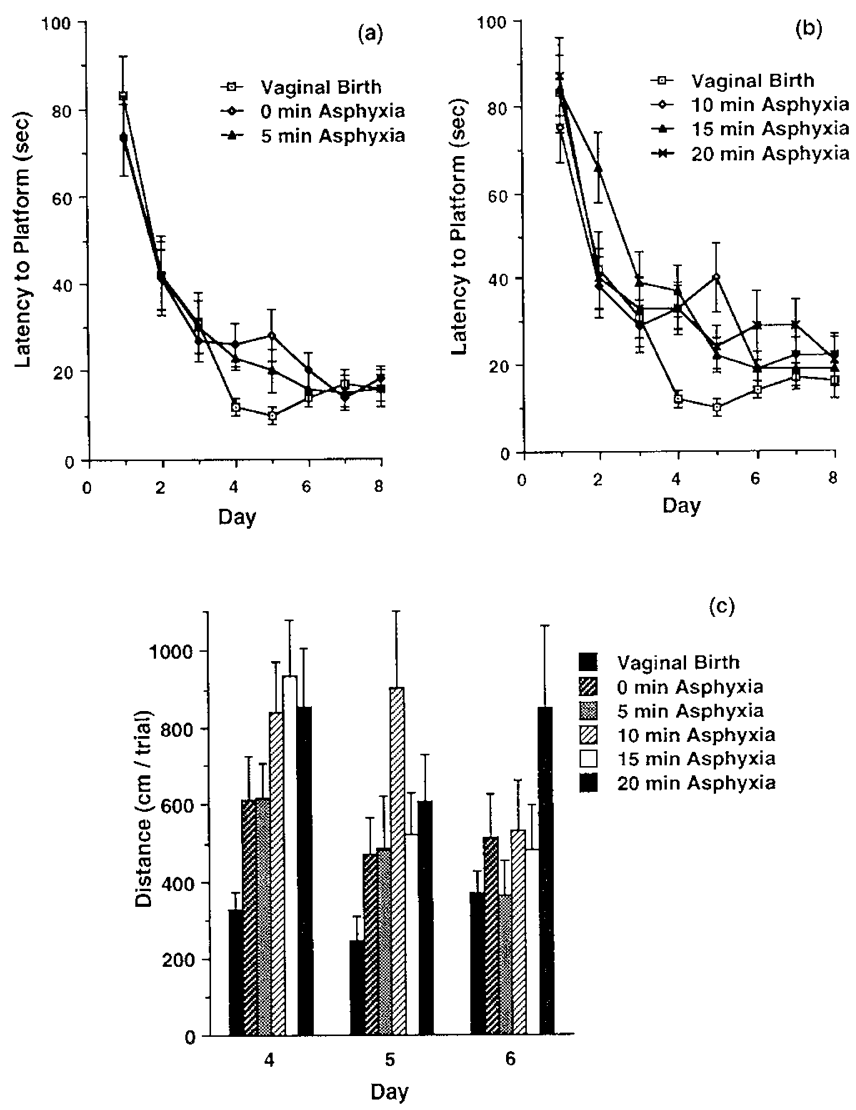

Figure 1. $a$ and $b$ Latency to mount a hidden platform in the Morris water maze for adult (4-mo-old) rats born vaginally or by cesarean section with 0,5 , 10,15 , or 20 min of birth asphyxia. Mean latency ( \pm SEM) is plotted as a function of day of testing. $n=10,15,12,13,15$, and 10 animals for groups born vaginally or by cesarean section with $0,5,10,15$, or 20 min of birth asphyxia, respectively. $c$, Corresponding swim distances on test d 4, 5, and 6 for the experiment shown in $a$ and $b$. 
5 min of birth asphyxia showed no significant deficit in the spatial learning task on any day of testing, in comparison with vaginally born controls.

Several observations indicate that asphytic animals showed no deficit in swimming ability or motivation in the water maze. Analysis of swimming distances on test $\mathrm{d} 4,5$, and 6 (Fig. 1c) showed a significant group effect $[F(5,142)=4.81, p<$ $0.0005]$ and indicated that adult rats that had undergone 10,15 , or $20 \mathrm{~min}$ of birth asphyxia spent their increased time in the pool by swimming further than did the vaginally born animals $(p<0.01)$. As a result, the swimming speed of adult rats that had undergone 10,15 , or 20 min of birth asphyxia was similar to that of vaginally born animals, on test $\mathrm{d} 4,5$, and 6 (with the exception of the 10-min group on d 5) (Table 2), indicating no impairment in swimming ability. In addition, when the platform was left visible in the water maze, animals that had undergone birth asphyxia found the platform as quickly as did controls (Table 2).

At the end of $8 \mathrm{~d}$ of trials with the hidden platform, the platform was removed from the water, and each animal was placed in the maze for a 60 -s probe trial (Fig. 2). The relative proportion of time spent in each of the four quadrants was similar for all groups of animals, i.e. birth group $\times$ quadrant interaction: $F(12,135)=0.64, p=0.80$. All animals (i.e. both control and asphytic) spent the greatest amount of their time in the quadrant that used to contain the platform (training quadrant), and analysis of data for the training quadrant alone indicated that the groups did not differ in proportion of time spent in the training quadrant $[F(4,45)=0.71, p=0.59]$. This suggests that all animals had been using similar cues and/or search strategies to find the hidden platform.

At the end of $8 \mathrm{~d}$ of trials with a hidden platform plus the probe trial, animals were mass trained (eight trials per day for

Table 2. Effect of birth asphyxia on swim speed when locating a hidden platform and latency to find a visible platform in the Morris water maze

\begin{tabular}{|c|c|c|c|c|}
\hline & & \multicolumn{3}{|c|}{ Swim speed $(\mathrm{cm} / \mathrm{s})^{*}$} \\
\hline & & d 4 & d 5 & $\mathrm{~d} 6$ \\
\hline Vaginal birth & $(10)$ & $29 \pm 2$ & $29 \pm 3$ & $31 \pm 2$ \\
\hline (0-min asphyxia & (14) & $24 \pm 2 \%$ & $22 \pm 1 \div$ & $27 \pm 2$ \\
\hline 5-min asphyxia & (12) & $28 \pm 1$ & $27 \pm 2$ & $27 \pm 2$ \\
\hline 10-min asphyxia & (13) & $25 \pm 1$ & $22 \pm 1 \dagger$ & $30 \pm 2$ \\
\hline 15-min asphyxia & $(15)$ & $25 \pm 1$ & $26 \pm 1$ & $26 \pm 2$ \\
\hline \multirow[t]{3}{*}{ 20-min asphyxia } & $(10)$ & $27 \pm 1$ & $28 \pm 2$ & $33 \pm 1$ \\
\hline & & \multicolumn{3}{|c|}{ Latency to platform $(s) \S$} \\
\hline & & d 1 & $\mathrm{~d} 2$ & d 3 \\
\hline Vaginal birth & (16) & $17 \pm 2$ & $10 \pm 1$ & $10 \pm 1$ \\
\hline 0 -min asphyxia & (9) & $13 \pm 2$ & $14 \pm 3$ & $8 \pm 1$ \\
\hline 5-min asphyxia & (12) & $15 \pm 2$ & $9 \pm 2$ & $10 \pm 1$ \\
\hline 10-min asphyxia & (6) & $15 \pm 3$ & $14 \pm 3$ & $10 \pm 2$ \\
\hline 15-min asphyxia & $(10)$ & $13 \pm 2$ & $11 \pm 2$ & $7 \pm 1$ \\
\hline 20-min asphyxia & (7) & $16 \pm 4$ & $13 \pm 2$ & $11 \pm 2$ \\
\hline
\end{tabular}

$n=$ numbers in parentheses.

* The $\mathrm{F}$ ratio for the main effect of birth group was $F(5,142)=3.85, p<$ 0.003 .

$\dagger p<0.05$.

$\ddagger p<0.01$, difference from vaginal birth value.

$\S$ The $F$ ratio for the main effect of birth group was $F(5,114)=0.88, p=$ 0.50 .

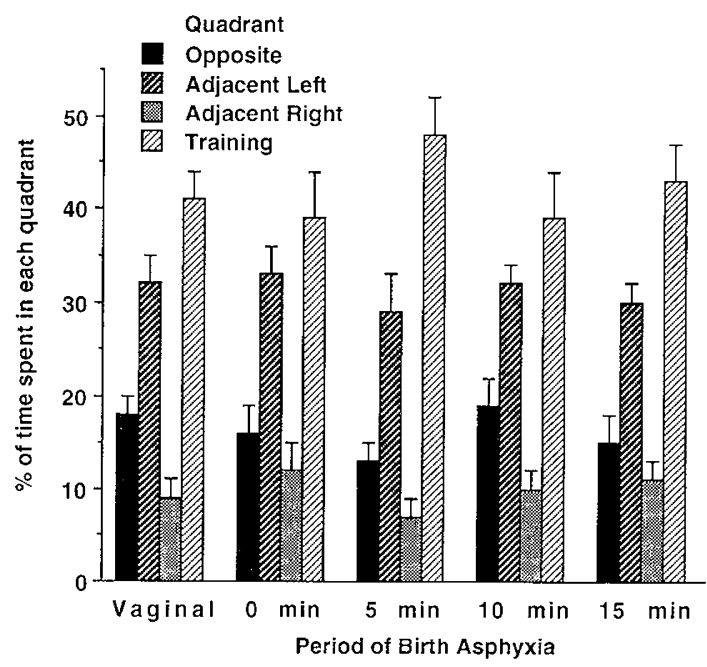

Figure 2. Proportion of time spent in each quadrant during a probe trial in the Morris water maze for adult rats born vaginally or by cesarean section with various periods of birth asphyxia. Quadrants are described by position in relation to the training quadrant. $n=10,11,10,8$, and 11 animals for groups born vaginally or by cesarean section with $0,5,10$, or 15 min of birth asphyxia, respectively.

$2 \mathrm{~d}$ ) to ensure that all had effectively learned the location of the hidden platform. The animals were left in their home cages for $1 \mathrm{wk}$ and then retested in the water maze for $4 \mathrm{~d}$. On retest after the $1 \mathrm{wk}$ of rest, there was no significant effect of birth group $[F(4,93)=1.26, p=0.29]$, indicating that control (vaginally born and 0 -min asphyxia) and asphytic (5, 10, and $15 \mathrm{~min}$ of asphyxia) rats found the hidden platform equally quickly (Fig. 3).

Separate experiments tested the water maze performance of adolescent (1.5 mo of age) rats that had undergone birth asphyxia. At $1.5 \mathrm{mo}$ of age, adolescent rats that had undergone

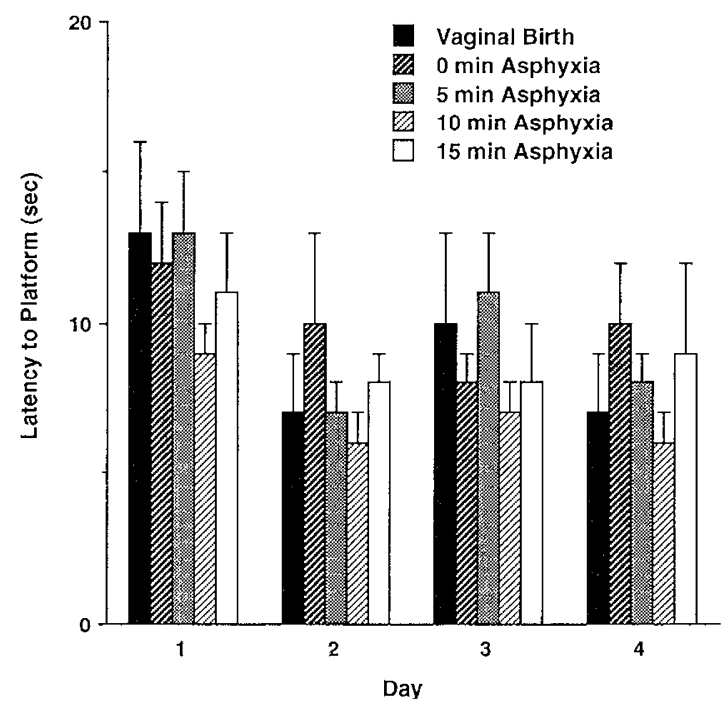

Figure 3. Retention/relearning of the Morris water maze task after 1 wk of rest. Adult rats that had been born vaginally or by ccsarean section with various periods of birth asphyxia were overtrained to find a hidden platform in the Morris water maze, allowed $1 \mathrm{wk}$ of rest, and then retested. Mcan latency ( \pm SEM) to mount the hidden platform on retest is shown as a function of day of testing. $n=10,10,10,8$, and 11 animals for groups born vaginally or by cesarean section with $0,5,10$, or $15 \mathrm{~min}$ of birth asphyxia, respectivcly. 
15 min of birth asphyxia showed no difference in latency to find the hidden platform in the Morris water maze compared with rats born vaginally or by cesarean section with 0 min of asphyxia (Fig. $4 a$ ) [ $F$ ratios for effects of birth group: $F(2,87)$ $=0.58, p=0.56$, and of day: $F(7,609)=39.91, p<0.00001$, group $\times$ day interaction: $F(14,609)=1.47, p=0.12]$. Compared with vaginally born animals, there was a tendency to increased latency for animals that had undergone $15 \mathrm{~min}$ of birth asphyxia on $\mathrm{d} 2-7$, but this did not reach significance.

A subgroup of animals that had received $8 \mathrm{~d}$ of trials with the hidden platform in the swim maze at 1.5 mo were retested using the same paradigm at 4 mo of age (Fig. 4b). On d 1 of testing, these animals showed a decreased latency to find the platform in comparison with 4-mo-old (compare Fig. $4 b$ with Fig. $1 a$ and $b$ ) animals naive to the swim maze. This was true for both control animals (vaginally born, $p<0.01$, and 0 -min asphyxia, $p<0.01$ ) and animals that had undergone $15 \mathrm{~min}$ of birth asphyxia $(p<0.01)$. This decreased initial latency lessened the likelihood of observing differences between control and asphytic animals on subsequent test days. Nonetheless, in the group of animals tested at $1.5 \mathrm{mo}$ and retested at 4 mo of age, animals that had undergone $15 \mathrm{~min}$ of birth asphyxia showed a significantly increased latency to find the platform, compared with either vaginally born or cesarean-sectioned $(0$-min asphyxia) controls, on $\mathrm{d} 5$ of the 4-mo retest $(p<0.05)$ (Fig. 4b).

Vaginally born animals, animals delivered by cesarean section with no asphyxia ( 0 -min asphyxia group), and animals that had undergone 15 min of birth asphyxia were also tested as adults ( 3 mo of age) for spontaneous alternation in a $\mathrm{T}$ maze, as well as for six other measures of sensorimotor function. There were no group differences in rates of spontaneous alternation or in measures of orientation, head scans, limb clasping, limb placing, or time to turn on an inclined grid (data not shown). The only group difference found was in a test of tactile stimulation. Animals that had undergone 15 min of birth asphyxia showed a significantly $(p<0.01)$ increased latency to begin grooming and to remove an adhesive label from the snout, compared with the 0 -min asphyxia group [for rats born vaginally or by cesarean section with 0 or 15 min of birth asphyxia, latencies (seconds) to begin grooming were $101 \pm 9$, $87 \pm 8$, and $121 \pm 8$, respectively; latencies (seconds) to remove the label were $109 \pm 9,92 \pm 7$, and $127 \pm 8$, respectively; means \pm SEM from 15 animals per group]. Once grooming began, animals in the 15-min group removed the label as quickly as did the 0-min group.

\section{DISCUSSION}

The main finding of this study is that rats that had undergone 10,15 , or $20 \mathrm{~min}$ of asphyxia during a cesarean birth, exhibited deficits in acquisition of a spatial learning task, as adults, in comparison with vaginally born animals. By contrast, no such deficit was detectable in rats that had been delivered by rapid cesarean section with no added period of asphyxia or with a 5 -min period of asphyxia. The threshold of $10 \mathrm{~min}$ of asphyxia for detection of deficits depends, to some extent, on the sensitivity of the spatial learning paradigm used. Nonetheless, it is
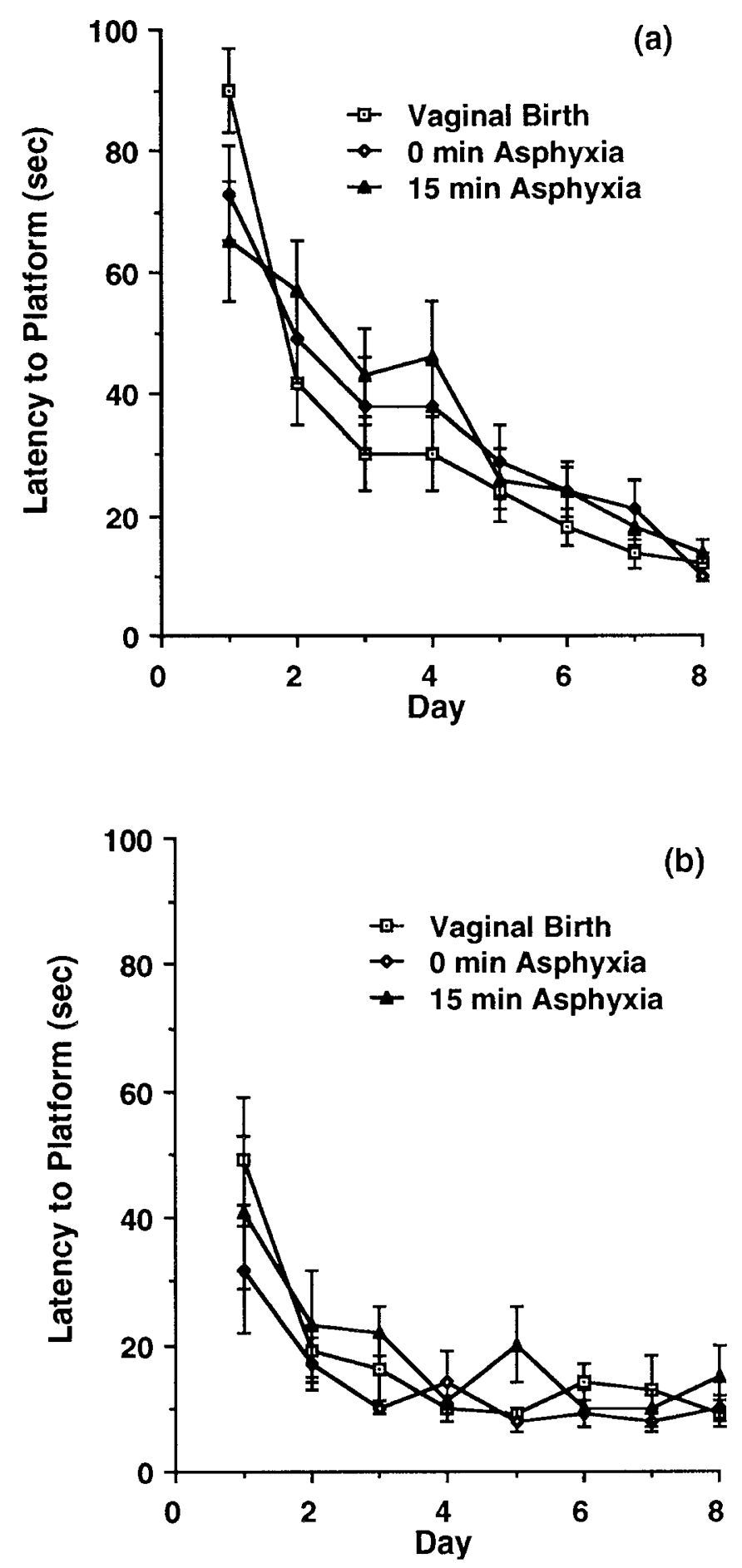

Figure 4. a, Latency to mount a hidden platform in the Morris water maze for adolescent (1.5 mo old) rats that were born vaginally or by cesarcan section with 0 or $15 \mathrm{~min}$ of birth asphyxia. Mean latency ( \pm SEM) is plotted as a function of day of testing. $n=16,15$, and 14 animals for groups born vaginally or by cesarean section with 0 or 15 min of birth asphyxia, respectively. $b$, Retention/relearning of the Morris water maze task at 4 mo of age. A subgroup of rats tested at $1.5 \mathrm{mo}$ in $a$ were retested in the same task at 4 mo. Mean latency $( \pm$ SEM) to mount the hidden platform on retest is plotted as a function of day of testing. $n=9,6$, and 6 animals for groups born vaginally or by cesarean section with 0 or 15 min of birth asphyxia, respectively.

of interest that fetal heart rate, in a very similar model of birth asphyxia, has been shown to be well maintained for 5 min of asphyxia, but to decline during 10-15 min of asphyxia, 
whercas arterial $\mathrm{O}_{2}$ saturation fluctuates throughout a 15 -min period of asphyxia (13). The conditions of water maze testing used in the present study, i.e. only two trials per day, with a relatively long intertrial interval of $15 \mathrm{~min}$, tend to increase sensitivity of the task for detection of deficits (23). Thus the deficits shown by the asphytic animals in this task are relatively subtle. Although effects of acute hypoxia/ischemia on the immature CNS have been studied to some extent at the cellular level, long-term behavioral effects of short periods of birth anoxia have received much less attention. However, use of behavioral measures may, in some cases, reveal that the CNS is more sensitive to anoxia than had been previously thought. In this regard, deficits in place learning in the Morris water maze have been reported in adult rats after transient occlusion of the common carotid arteries ("two-vessel occlusion" model) in the absence of observable histologic CNS damage (24).

In the present study, the cesarean procedure alone did not produce a significant deficit in spatial learning. However, there was a tendency for increased acquisition latencies in the 0-min asphyxia group compared with vaginally born controls, suggesting that the cesarean procedure alone may produce subthreshold effects on spatial learning. Anoxia, in combination with the cesarean procedure, resulted in significant spatial learning deficits. In fact, comparison between period of asphyxia and extent of spatial learning deficit reveals a quasidose-effect relationship. Compared with vaginally born animals, the 5-min asphyxia group showed no significant deficit in spatial learning and the 10-min group showed a deficit on $\mathrm{d} 4$ and 5 of testing but no overall group effect, whereas the 15- and 20 -min groups showed significant effects on comparison of latencics across the $\delta \mathrm{d}$ of testing. This finding is consistent with the notion that the length of the period of asphyxia during the cesarean section procedure determined the degree of spatial lcarning deficits observed. In comparison with the adult nervous system, immature nervous tissue from a number of species is known to be more resistant to hypoxia/ischemia (25). Thus, it is not surprising that near normal acquisition in place learning was seen in adult rats that had undergone 5 min of complete anoxia during birth and long-term deficits were seen only with longer periods of anoxia.

Global birth asphyxia may potentially affect any organ sys$\mathrm{tcm}$ or tissue in the body. Thus increased latencies in the Morris maze on the part of asphytic animals could have been due to deficits in locomotor ability and/or motivation to find and mount the hidden platform. However, this did not appear to be the case. Animals that had undergonc up to $20 \mathrm{~min}$ of birth anoxia showed no systematic decrease in swim speed and mounted a visible platform in the water maze as quickly as did vaginally born animals. The latter result with the visible platform also indicates that asphytic animals had no gross visual abnormalitics, an important concern because, at least in humans, perinatal hypoxia/ischemia is often associated with $\mathrm{vi}^{-}$ sual disturbance (12). In addition, as assessed by quadrant time during a probe trial in the swim maze, animals that had undergone birth asphyxia secmed to use a similar strategy to find the hidden platform as did vaginally born animals.
Adult rats that had undergone 15 min of birth asphyxia performed similarly to control animals (vaginal birth or cesarcan section with 0 min of asphyxia) in a variety of sensorimotor tests and showed similar rates of spontaneous alternation in a $\mathrm{T}$ maze, supporting our gross observations that behavioral deficits do not secm to be widespread or severe in the asphytic animals. The one sensorimotor task in which asphytic animals showed a slight impairment was in latency to initiate facial grooming when a sticky label was placed on the snout; this raises the possibility that asphytic animals may show minor defects in sensory attention, that could, in turn, contribute to decreased spatial learning ability. Once grooming began in response to the sticky label, asphytic animals took no longer to remove the label than did controls, indicating that asphytic animals retain the fine motor ability to complete this maneuver.

The deficits in spatial learning observed after birth asphyxia in the present study may result from direct tissue injury sustained during the period of birth asphyxia. The localization of the CNS pathology, if any, that is responsible for the deficit in spatial lcarning after birth asphyxia remains to be determined. Deficits in spatial learning in the Morris water maze have been reported after circumscribed lesions to the hippocampus and related structures $(17-19,26,27)$, as well as other structures, such as frontal or parietal cortex (28), medial caudate-putamen (29), and the nucleus basalis magnocellularis (23). Involvement of damage to hippocampal structures in the spatial learning deficits produced by birth asphyxia in the present study is likely in view of the noted vulnerability of the hippocampus to ischemia/hypoxia and the observation by Bjelke et al. (13) of a modest loss of neurons in hippocampal CA1 and CA3 regions in a very similar model of birth asphyxia. One may also consider the possibility that birth asphyxia might damage a selectively vulnerable subpopulation of neurons within a given brain region. For example, spatial learning deficits are reported to occur after combined cholinergic/serotonergic denervation of the forcbrain (30) or specific $N$-methyl-D-aspartate receptor blockade (31).

Alternatively, the observed deficits in spatial learning in the present study may not be due to direct tissue injury during birth asphyxia but may result from events occurring secondary to the initial asphytic event. For cxample, the decreased weights observed in young animals that had undergone birth asphyxia suggest that early postnatal feeding may be suboptimal in asphytic animals due to impaired feeding ability and/or competition for maternal feeding time with larger, fitter litter mates. Thus, birth asphyxia may cause animals to have an altered nutritional status that secondarily affects maze learning ability. Early caloric restriction, particularly during the period before weaning in the rat, has been documented to produce permanent deficits in body and brain weight as well as reductions in cell numbers and lipid content in the brain $(32,33)$. Detailed information concerning specific long-term cognitive effects, if any, associated with varying degrees of early undernourishment in the rat, is lacking, although both undernourishment and overnourishment have been reported to produce altered behavioral responses, accompanied by morphologic changes in CNS, in some animals models (33-36). Another secondary factor that may influence maze performance in asphytic animals is the 
stress response. Recent studies in our laboratory have documented that animals undergoing asphyxia during a cesarean section exhibit long-term alterations in glucocorticoid secretion basally and in response to acute stress (Boksa P, Krishnamurthy $\mathrm{A}$, Sharma S, Wilson $\mathrm{D}$, manuscript in preparation), as well as alterations in central monoamine metabolism after repeated stress (Brake W, Noel M, Boksa P, Gratton A, manuscript in preparation). Altered responsiveness to the novelty or stress inherent in swim maze testing and/or chronically altered responsiveness to daily stressors could conceivably impinge on performance in the swim maze. However, regardless of which secondary mechanisms intervene, the primary event responsible for spatial learning deficits in our experiments is clearly the period of birth anoxia during cesarean section.

Another important issue addressed in this study was whether spatial learning deficits in animals that had undergone birth asphyxia became worse or resolved during the progression from adolescence to adulthood in comparison with agematched controls. Our results showed that 1.5-mo-old (adolescent) animals that had undergone $15 \mathrm{~min}$ of birth asphyxia showed no significant deficit in spatial learning compared with 1.5-mo-old control animals. However, significant deficits in spatial learning were observed in a separate group of 4-mo-old (adult) animals that had undergone $10-20 \mathrm{~min}$ of birth asphyxia compared with their age-matched controls. Inspection of the data suggests that this age-dependent deficit in asphytic compared with vaginally born animals is not due to an early neurodegenerative process in the asphytic animals, becaue performance of asphtyic animals does not, in fact, worsen with age. Rather the data are consistent with the interpretation that animals undergoing birth asphyxia show an impairment in the development of improved spatial learning abilities normally observed in control animals. This interpretation is supported by comparison of the spatial learning data from 1.5- and 4-mo-old control animals (compare vaginal birth groups in Figs. $1 a$ and $4 a$, i.e. data from separate groups of animals tested at either 1.5 or 4 mo of age). This comparison suggests that the performance of control animals in the spatial learning task improves with age from 1.5 to $4 \mathrm{mo}$. By contrast, latencies for animals that had undergone $15 \mathrm{~min}$ of birth asphyxia were very similar at 1.5 and 4 mo of age (compare 15-min asphyxia groups in Figs. $1 b$ and $4 a$ ), showing little tendency to improved performance with age.

Regardless of whether the mechanism involves a neurodevelopmental or a neurodegenerative process, the data clearly show that, in comparison with their age-matched controls, asphytic animals showed no deficit in spatial learning at $1.5 \mathrm{mo}$ of age, whereas a deficit could be measured at 4 mo. This conclusion is also supported by spatial learning data from a subgroup of the 1.5 -mo-old animals that were grown to 4 mo of age and retested (Fig. 4b). On retest, a significant impairment in place learning was still observed on $\mathrm{d} 5$ of testing, even though these animals had received prior training at $1.5 \mathrm{mo}$.

Adult animals that had undergone $10-20 \mathrm{~min}$ of birth asphyxia showed only a transient deficit in spatial learning and effectively learned the position of the hidden platform in the water maze by d 8 of testing. After overtraining, short-term (1 wk) retention of the platform position was also similar in control and asphytic animals. The spatial learning data from 1.5-mo-old animals that were retested at 4 mo of age showed that latencies on $\mathrm{d} 1$ of retest for both control and asphytic animals were less than d-1 latencies recorded for 4-mo-old animals naive to the water maze. This indicates that both control animals and animals that had undergone $15 \mathrm{~min}$ of birth asphxia are able to retain some element of learning over a longer course of $2.5 \mathrm{mo}$.

In closing, we note that the CNS of the human newborn is thought to be at a more mature developmental stage than that of the rat at birth, with the brain of a rat at approximately postnatal d 10 resembling the accelerated period of synaptogenesis, myelinization, and astrocyte proliferation characteristic of the human newborn CNS at term (37-39). However, use of postnatal rat models does not allow for examination of the consequences of global asphyxia on the entire animal, calling into play all of the reflex and compensatory mechanisms specific to the time of birth. The advantage of the rat model of birth asphyxia for studies of long-term behavioral effects lies in the ability to reproduce these birth factors in their entirety in a species that readily lends itself to later behavioral testing (40). The model should also prove useful for characterization of the pattern of morphologic and biochemical alterations resulting from global anoxia at birth and may aid in the development of therapeutic interventions designed to minimize resulting CNS damage. However, inasmuch as parallels can be drawn between rat models and clinical human events, the rat model of birth asphyxia may relate, in some respects, more to the condition of the premature rather than the term human infant. In this regard, it is of note that, in comparison with term human infants, prematurely born human infants experience birth anoxia much more frequently, whereas later deficits in school performance are also more often encountered in the premature group $(2,3,41)$. However, even in the term human infant undergoing moderate asphyxia, academic underachievement as well as long-term deficits in specific tests of attention and short-term recall have recently been documented (4).

Acknowledgments. The authors thank Sharon Welner and Michael Meaney for helpful discussion and Alain Gratton and Joseph Rochford for critically reading the manuscript.

\section{REFERENCES}

1. Brann Jr AW 1986 Hypoxic ischemic encephalopathy (asphyxia). Pediatr Clin North Am 33:451-464

2. Vannucci RC 1990 Experimental biology of cercbral hypoxia-ischemial relation to perinatal brain damage. Pediatr Res 27:317-326

3. Volpe JJ 1992 Brain injury in the premature infant: current concepts of pathogenesis and prevention. Biol Neonate 62:231-242

4. Robertson CMT, Finer NN 1993 Long-term follow-up of term neonates with perinatal asphyxia. Clin Pcrinatol 20:483-499

5. Dawes GS, Jacobson HN, Mott JC, Shelley HJ 1960) Some observations on foetal and new-born rhesus monkeys. J Physiol (Lond) 152:271-298

6. Dawes GS, Mott JC, Shelley HJ 1959 The importance of cardiac glycogen for the maintenance of life in foetal lambs and new-born animals during anoxia. J Physiol (Lond) $146: 516-538$

7. Vannucci RC, Duffy TE 1976 Carbohydrate metabolism in fetal and neonatal rat brain during anoxia and recovery. Am J Physiol 230:1269-1275

8. Jones CT 1980 Circulating catecholamines in the fetus, their origin, actions and significance. In: Parvez H, Parvez S (cds) Biogenic Amines in Development. Elsevier, Amsterdam, pp 63-86

9. Kudlacz EM, Navarro HA, Slotkin TA 1989 Phosphatidic acid phosphatase in neonatal rat lung: effects of prenatal dexamethasone or terbutaline treatment on basal 
activity and on responsiveness to beta adrenergic stimulation. J Pharmacol Exp Ther 250):236-240

10. Lagercrant $\mathrm{H}$, Bistoletti P 1973 Catecholamine release in the newborn infant at birth Pediatr Res 11:889-893

11. Lagercrantz H. Slotkin TA 1986 The "stress" of being born. Sci Am 254:100-107

12. Shankaran S, Woldt E, Koepke T, Bedard MP, Nandyal R 1991 Acute neonatal morbidity and long-term central nervous system sequelae of perinatal asphyxia in term infants. Early Ilum Dev 25:135-148

13. Bjeike B, Andersson K, Ogren SO, Bolme P 1991 Asphytic lesion: proliferation of tyrosine hydroxylase-immunoreactive nerve cell bodies in the rat substantia nigra and functional changes in dopamine transmission. Brain Res 543:1-9

14. Williams CE, Gunn AJ, Synck B, Gluckman PD 1990) Delayed seizures occurring with hypoxic-ischemic encephalopathy in the fetal shecp. Pediatr Res 27:561-565

15. Hill A, Volpe JJ 1981 Scizures, hypoxic-ischemic brain injury, and intraventricular hemorrhage in the newborn. Am Neurol 10:109-121

16. Mulligan JC, Painter MJ, O'Donoghue PA, McDonald HM, Allen AC, Taylor PM 1980 Neonatal asphyxia. II. Neonatal mortality and long-term sequelae. J Pediatr $96: 903-907$

17. Morris RGM, Garrud P, Rawlins JNP, O’Keefe J 1982 Place navigation impaired in rats with hippocampal lesions. Nature 297:681-683

18. Moser E, Moser M-B, Andersen P 1993 Spatial learning impairment parallels the magnitude of dorsal hippocampal lesions, but is hardly present following ventral lesions. J Neurosci 13:3916-3925

19. Sutherland RJ, Whishaw IQ, Kolb B 1983 A behavioural analysis of spatial local ization following electrolytic, kainate- or colchicinc-induced damage to the hippocampal formation in the rat. Behav Brain Res 7:133-135

20. Dunnet! SB, Whishaw IQ, Jones GH, Bunch ST 1987 Behavioral, biochemical and histochemical effects of different neurotoxic amino acids injected into nucleus basalis magnocellularis of rats. Neuroscience 20:653-669

21. Schallert T, Upchurch M, Lobaugh N, Farrar SB, Spirduso WW, Gilliam P, Vaughn D, Wilcox RE 1982 Tactile extinction: distinguishing between sensorimotor and motor asymmetries in rats with unilateral nigrostriatal damage. Pharmacol Biochem Bchav 16:455-462

22. Welner SA, Koty ZC 1993 Amelioration of sensory attention and sensorimotor deficits by chromaffin cell grafts to the cercbral cortex of nucleus basalis magnocellularis lesioned rats. Behav Brain Res 59:73-81

23. Mandel RJ, Gage FH, Thal $[J 1989$ Enhanced detection of nucleus basalis magnocellularis lesion-induced spatial learning deficit in rats by modification of training regimen. Behav Brain Res 31:221-229

24. Jaspers RMA, Block F, Hejm C, Sontag K-H 1990 Spatial learning is affected by transicnt occlusion of common carotid arteries (2VO): comparison of bchavioural and histopathological changes after "2VO" and "four-vessel-occlusion" in rats. Neurosci Lett 117:149-153

25. Khabat $\mathrm{H} 1970$ The greater resistance of very young animals to arrest of the brain circulation. Am J Physiol 130:588-599
26. Morris RGM, Schenk F, Tweedie F, Jarrard LE 1990) Ibotenate lesions of hippocampus and/or subiculum: dissociating components of allocentric spatial lcarning. Eur $\mathrm{J}$ Neurosci 2:1016-1028

27. Netto CA, Hodges H, Sinden JD, Le Peillet E, Kershaw T, Sowinski P, Mieldrum BS, Gray JA 1993 Effects of fetal hippocampal ficld grafts on ischemia-induced deficits in spatial navigation in the water maze. Neuroscience 54:69-92

28. Kolb B, Sutherland R.J, Whishaw IQ 1983 A comparison of the contributions of the frontal and parietal association cortex to spatial localization in rats. Behav Neurosci 97:13-27

29. Whishaw IQ, Mittleman G, Bunch ST, Dunnett SB 1987 Impairments in the acquisition, retention and selection of spatial navigation strategies after medial caudateputamen lesions in rats. Behav Brain Res 24:125-138

30. Nilsson OG, Strecker RE, Daszuta A, Bjorklund A 1988 Combined cholinergic and scrotonergic dencrvation of the forebrain produces severe deficits in a spatial learning task in the rat. Brain Res 453:235-246

31. Davis S, Butcher SP, Morris RGM 1992 The NMDA receptor antagonist D-2-amino5-phosphonopentanoate (D-AP5) impairs spatial learning and LTP in vivo at intracerebral concentrations comparable to those that block LTP in vitro. J Neurosci $12: 21-34$

32. Dobbing J, Sands J 1971 Vulnerability of developing brain. IX. The effect of nutritional growth retardation on the timing of the brain growth spurt. Biol Nconate 19:363-378

33. Winick M 1983 Nutrition and mental development. Med Clin North Am 54:14131429

34. Brock JW, Prasad C. 1991 Motor, but not sensory, cortical potentials are amplified by high protein diet. Physiol Behav 50:887-893

35. Brock JW, Prasad C 1992 Alterations in dendritic spinc density in the rat brain associated with protein malnutrition. Dev Brain Res 66:266-269

36. Smart JL, Dobbing J 1971 Vulnerability of developing brain. VI. Relative effects of foetal and early postnatal undernutrition on reflex ontogeny and development of behaviour in the rat. Brain Res 33:303-314

37. Dobbing J, Sands J 1979 Comparative aspects of brain growth spurt. Early Hum Dev $3: 79-83$

38. Jilek L, Travnickova E, Trojan S. 1970 Characteristic metabolic and functional responses to oxygen deficiency in the central nervous system. In: Stave U (cd) Physiology of the Perinatal Period: Vol. 2, Functional and Biochemical Development in Mammals. Appleton-Century Crofts, Meredith Corp., New York, pp 987-1041

39. Romign HJ, Hofman MA, Gramsbergen A 1991 At what age is developing rat cortex comparable to that of the full term human baby? Early Hum Dev 26:61-67

40. Raju TK 1992 Some animal models for the study of perinatal asphyxia. Biol Neonate $62: 202-214$

41. Jongmans M, Henderson S, de Vries L, Dubowitz L 1993 Duration of periventricular densities in preterm infants and neurological outcome at 6 years of age. Arch Dis Child 69:9-1.3 